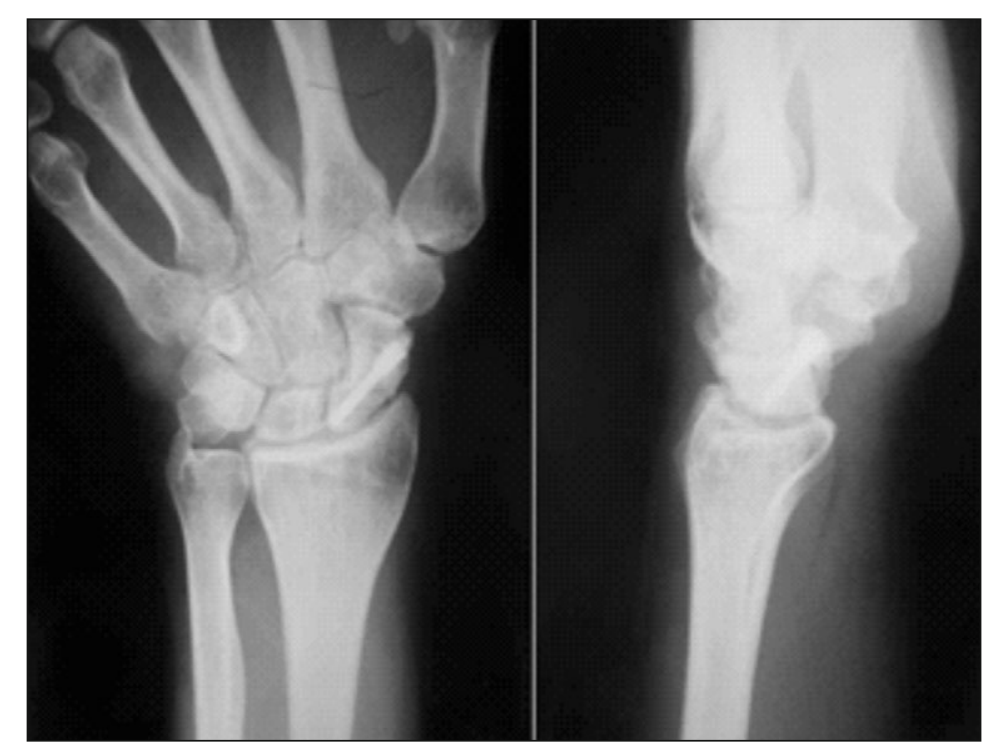

Fig. 3. Controllo radiografico a 16 mesi

\section{Letture consigliate}

Chang SH, Hsu YM, Wang YJ et al (2009) Fabrication of pre-determined shape of bone segment with collagen-hydroxyapatatite

scaffold and autogenous plateletrich plasma. J Mater Sci Mater Med 20:23-31 (2007) Enhanced tibial osteotomy healing with use of bone



Fig. 4. Controllo clinico a 16 mesi

Dallari D, Savarino L, Stagni C et al grafts supplemented with platelet gel and bone marrow stromal cells. J Bone Joint Surg Am 89:2413-2420

Paoloni J, De Vos RJ, Hamilton B et al (2011) Platelet-rich plasma treat- ment for ligament and tendon inPeerbooms JC, Sluimer J, Bruijn DJ, Gosens T (2010) Positive effect of an autologous platelet concentrate in lateral epicondylitis in a juries. Clin J Sport Med 21:37-45 double-blind randomized controlled trial: platelet-rich plasma versus corticosteroid injection with 1-year follow up. Am J Sports Med 38:255-262

Sampson S, Gerhardt M, Mandelbaum B (2008) Platelet rich plasma injection grafts for musculoskeletal injuries: a review. Curr Rev Musculoskelet Med 1:165174

Taylor DW, Petrera M, Hendry M, Theodoropoulos JS (2011) A systematic review of the use of platelet-rich plasma in sports medicine as a new treatment for tendon and ligament injuries. Clin J Sport Med 21:344-352

Vincenzi D, Perna L, Riccipetitoni F Tomassini I (2008) Utilizzo di gel piastrinico autologo in un caso di grave perdita di sostamza ossea. Blood Transfus 6.374

Woodell-May J, Pietrzak W (2008) Platelet-rich plasma in orthopaedics. In: Pietrzak W (Ed.) Musculoskeletal tissue regeneration. Humana Press, Totova, NY:547568

\title{
Applicazioni biotecnologiche nella Clinica Ortopedica di Padova
}

\section{R. Aldegheri ${ }^{1}$, A. Pozzuoli²}

1Professore Ordinario di Ortopedia e Traumatologia, Università degli Studi di Padova;

¿Laboratorio Bio-Ortopedia, Clinica Ortopedica, Padova

DOI 10.1007/s10261-013-0052-3

\begin{abstract}
Biotechnology applications in the Orthopedic Clinic of Padua

We usually apply the newest and more accepted biotechnological surgical treatments for bone regeneration. Our results are similar to those in the literature. At the same time, in our Bio-Orthopedics Laboratory, we are conducting studies and research mainly on two topics: bone tissue engineering and reactions to metal release from total hip prostheses. We present in this paper our ongoing research and the preliminary results.
\end{abstract}

\section{Introduzione}

Nell'ultimo decennio abbiamo effettuato esperienza clinica con le più affermate tecniche chirurgiche riferibili alla biotecnologia. Le indicazioni sono state quelle consigliate dalla letteratura più accreditata, quali le fratture non consolidate e le perdite di sostanza ossea con e senza infezione. Contemporaneamente abbiamo anche effettuato ricerche innovative, sempre sullo stesso argomento, nel nostro Laboratorio Bio-Ortopedia diretto dalla dott.ssa Assunta Pozzuoli $\mathrm{MSc}$, in particolare nell'ambito della rigenerazione ossea e della reazione dei tessuti ai metalli.

\section{Rigenerazione ossea}

La rigenerazione ossea è un processo multifattoriale che richiede l'intervento di vari elementi, tra cui le cellule osteogeniche, fattori osteoinduttivi e uno "scaffold" osteoconduttivo. Tutti questi elementi vengono ripresi, singolarmente o in associazione, nei metodi tradizionali di trattamento che si basano sull'utilizzo degli innesti ossei, i quali possono essere di derivazione autologa, omologa, eterologa o sintetica. Tuttavia i limiti connessi a queste tecniche e la necessità di grandi quantità di osso hanno stimolato il nostro interesse a sviluppare nuove metodiche in grado di rigenerare un tessuto osseo strutturalmente e funzionalmente simile al normale [1].

Un approccio innovativo per il trattamento delle perdite ossee è stato l'ingegneria tissutale, basata sulle proprietà delle cellule staminali mesenchimali (hMSCs), cellule multipotenti in grado di migrare, proliferare e differenziare in senso osteoblastico in vitro, se seminate su un biomateriale adeguato e in appropriate condizioni di coltura La fonte più utilizzata di hMSCs è rappresentata dal midollo osseo (hBMSCs) ma, recentemente, grande interesse ha suscitato il tessuto adiposo, in quanto nella sua frazione stromale-vascolare è stata identificata una popolazione di cellule staminali mesenchimali (hASCs) con caratteristiche funzionali simili alle hBMSCs. Infatti, come le hBMSCs, le hASCs sono multipotenti e in grado di differenziarsi, in condizioni di coltura adeguate, in varie linee cellulari, ma presentano numerosi vantaggi quali la facilità e sicurezza di prelievo; una maggior resa rispetto alle hBMSCs (2\% delle cellule nucleate ottenute da lipoaspirato contro $0,001 \%$ delle cellule nucleate nel midollo osseo di un adulto sano); una maggior disponibilità, data l'abbondanza di tessuto adiposo; infine, la facilità di coltivazione ed espansione in vitro e una maggiore resistenza in coltura. Sulla base di questi presupposti abbiamo effettuato una sperimentazione con lo scopo di valutare in vitro le potenzialità differenziative in senso osteogenico delle hASCs e delle hBMSCs seminate in presenza di alcuni biomateriali osteoinduttivi (matric ossee demineralizzate, DBM) e osteoconduttivi (materiali ceramici) in uso nella pratica clinica. Inoltre, poiché le cellule staminali potrebbero essere implicate nel processo di oncogenesi, abbiamo posto particolare attenzione nella valutazione della presenza di eventuali alterazioni geniche che potrebbero compromettere la sicurezza del loro utilizzo. I risultati sin qui ottenuti confermano un simile potenziale differenziativo, in vitro, tra hBMSCs e hASCs. Lassenza di alterazioni geniche dimostra anche la sicurezza dei materiali delle metodiche utilizzate in vista di una loro potenziale applicazione in ambito clinico [2,3].

Il tessuto adiposo, quindi, rappresenta una fonte alternativa potenzialmente meno problematica rispetto al midollo osseo, di cellule osteoprogenitrici in chirurgia ortopedica: è di facile prelievo, comporta minimo "discomfort" per il paziente, è possibile ottenerne quantità elevate, ha una maggior resa rispetto al midollo osseo; il tempo necessario, i costi e il rischio di contaminazione cellulare sono inferiori. Speriamo che, quanto prima, si possano utilizzare in clinica.

Parallelamente alla sperimentazione in vitro, i biomateriali testati sono stati impiegati anche in ambito chirurgico. In particolare, alcune matrici ossee demineralizzate, utilizzate da sole o in associazione ad altri sostituti ossei, hanno portato a ottimi risultati, soprattutto nel trattamento delle pseudoartrosi e nel riempimento di cisti ossee, o encondromi, o "gap" ossei dopo fratture comminute [4].

\section{Rilascio di metalli da protesi}

Il secondo settore d'interesse dei nostri studi origina dal sempre più diffuso impiego di mezzi di sintesi e impianti protesici da cui possono conseguire numerose problematiche legate al rilascio di metalli nei pazienti portatori di impianti. Per questo, abbiamo in corso uno studio retrospettivo che si prefigge di indagare tutti i pazienti sottoposti a sostituzione dell'anca con protesi totali (metallo su metallo, ceramica su ceramica e TriboFit $^{\circledR}$ Hip System [5]) con l'obiettivo di in-

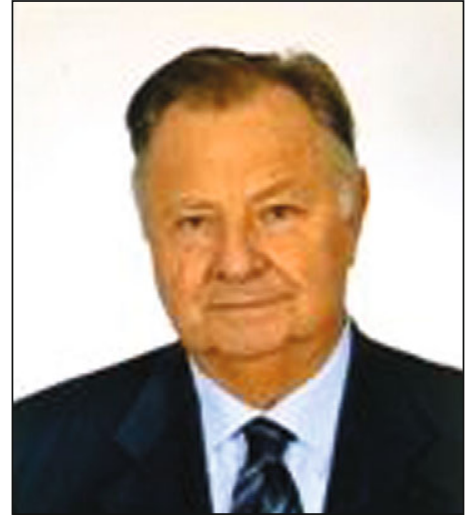

R. Aldegheri

dividuare precocemente i soggetti che hanno un medio-alto rilascio di cobalto e cromo nei liquidi biologici, in quanto a maggior rischio di sviluppare complicanze, sia locali (quali la mobilizzazione della protesi) sia sistemiche (danno d'organo), evitando e/o limitando così gravi conseguenze per il paziente stesso ed elevati costi per il Sistema Sanitario Nazionale.

\section{Bibliografia}

Franchini M, Dupplicato P, Ferro I et al (2005) Efficacy of platelet gel in reconstructive bone surgery. Orthopedics 28:161163

Pozzuoli A, Tamburin S, Zavan $B$ et al (2011) Bone regeneration from adipose tissue: experimental study. J Orthop Traumatol 12[Suppl.]:162

Pozzuoli A, Zamperetti M, Zavan B et al (2012) Osteogenesis from adipose tissue: an in vitro study. J Orthop Traumatol 12[Suppl.]:58-59

Carniel D, Pozzuoli A, Aldegheri R (2011) Osteoinductive capacity of demineralized bone matrix: a clinical study. J Orthop Traumatol 12[Suppl.]: 156-157

Berizzi A, Friso P, Pozzuoli A, Aldegheri R (2011) Our experience with Tribofit ${ }^{\circledR}$ Acetabular System. J Orthop Traumatol 12[Suppl.]:148-149 